\title{
Fluorine F 18 PARP Inhibitor
}

National Cancer Institute

\section{Source}

National Cancer Institute. Fluorine F18 PARP Inhibitor. NCI Thesaurus. Code C155292.

A radiotracer consisting of a $1(2 \mathrm{H})$ phthalazinone-based poly(ADP-ribose) polymerase 1 (PARP1) inhibitor (PARPi) radiolabeled with the positron emitting isotope fluorine F 18, which can potentially be used for the imaging of PARP1 expression using positron emission tomography (PET). Upon administration, fluorine F 18 PARPi targets and binds to PARP1. Upon PET, PARP1-expressing tumor cells can be visualized. PARP1, which is overexpressed in many cancer cell types, catalyzes post-translational ADP-ribosylation of nuclear proteins and plays a key role in the repair of DNA strand breaks. 